\title{
Reologia de concretos de alto desempenho aplicados na construção civil - Revisão
}

\section{(Review article: Rheology of high performance concretes applied in building site)}

\author{
A. L. de Castro ${ }^{1}$, J. B. L. Liborio' ${ }^{2}$, V. C. Pandolfelli ${ }^{1}$ \\ ${ }^{I}$ Departamento de Engenharia de Materiais, Universidade Federal de S. Carlos, Rod. Washington Luiz, km 235, \\ S. Carlos, SP 13565-905 \\ ${ }^{2}$ Escola de Engenharia de S. Carlos, Universidade de S. Paulo, Av. Trabalhador Sancarlense 400, Centro, \\ S. Carlos, SP 13566-590 \\ alelorencastro@yahoo.com.br,vicpando@power.ufscar.br
}

\begin{abstract}
Resumo
Do ponto de vista reológico, o concreto pode ser entendido como uma concentração de partículas sólidas em suspensão (agregados) em um líquido (pasta de cimento). Sendo assim, seu comportamento no estado fresco deve ser estudado a partir dos conceitos da reologia, ciência voltada para o estudo das deformações e escoamento de um fluido sob a influência de tensões. As pesquisas efetuadas ao longo do tempo resultaram em uma série de equações que descrevem o comportamento do concreto fresco. Sabe-se que o comportamento reológico desse material se aproxima de um fluido binghamiano, sendo necessários dois parâmetros para sua caracterização: a tensão de escoamento e a viscosidade plástica. A reologia do concreto fresco tem sido estudada com determinações que variam entre métodos de ensaio simples e práticos, como o ensaio de abatimento de tronco de cone, até equipamentos mais sofisticados que determinam as curvas de cisalhamento do material, como os reômetros. Dessa maneira, é possível determinar a relação entre a tensão de cisalhamento e a taxa de cisalhamento sob condições definidas fisicamente. Como o concreto fresco é um material heterogêneo, uma aproximação mais precisa do seu comportamento reológico é dada pela análise direta das forças (torque) que resultam do cisalhamento (velocidade de rotação) do concreto. Assim, o presente artigo apresenta uma revisão bibliográfica sobre os principais conceitos relacionados com a reologia de concretos de alto desempenho aplicados na construção civil, envolvendo desde sua caracterização reológica até os métodos de ensaio utilizados para a determinação das propriedades reológicas.

Palavras-chave: reologia, tensão de escoamento, viscosidade plástica, concreto de alto desempenho.
\end{abstract}

\begin{abstract}
From a rheological point of view, concrete can be understood as a concentrate suspension of solid particles (aggregates) in a viscous liquid (cement paste). In such case, its fresh behavior should be evaluated based on the concepts of rheology, the science which relates the deformation and flow of a fluid under stress influence. Several studies had already been accomplished along the time, resulting in a series of equations that describe the fresh concrete behavior. It is known that the rheological behavior of this material is close to that of a Bingham fluid and two rheological parameters are required for its description: yield stress and plastic viscosity. The rheology of fresh concrete has been studied by measurements that vary among simple and practical test methods, such as the slump test, and more sophisticated equipments that determine the flow curve of the material, such as the rheometer. Therefore, it is possible to attain a correlation between shear stress and shear rate under conditions defined physically. As fresh concrete is a heterogeneous material, a more accurate approach of its rheological behavior is given by the direct analysis of the forces (torque) that result from the shear (rotation speed) of the concrete. Thus, this paper presents a review concerning the main concepts related with the rheology of high performance concretes applied in building site, which involves the rheological characterization and the test methods used for the measurement of the rheological properties.
\end{abstract}

Keywords: rheology, yield stress, plastic viscosity, high performance concrete.

\section{INTRODUÇÃO}

O concreto é considerado um material composto, tendo como componentes principais o cimento, os agregados e a água. No caso dos concretos de alto desempenho, aditivos químicos e compostos minerais são incorporados à mistura "tradicional" para que uma variedade de propriedades e características seja obtida. Do ponto de vista reológico, esse material pode ser entendido como uma concentração de partículas sólidas em suspensão (agregados) em um líquido viscoso (pasta de cimento). Em uma escala macroscópica, o concreto fresco flui como um líquido [1].

Termos como trabalhabilidade, consistência, capacidade de escoamento, mobilidade e capacidade de bombeamento 
têm sido usados para descrever o comportamento do concreto no estado fresco; porém, esses termos refletem mais pontos de vista pessoais do que precisão científica [2]. Assim, sendo o concreto um líquido, nada mais adequado do que aplicar os conceitos da reologia ao estudo do seu comportamento quando no estado fresco. As propriedades reológicas do concreto fresco, em particular dos concretos de alto desempenho, são importantes principalmente no período em que o material é lançado nas fôrmas e podem ser determinadas em qualquer momento durante o período de indução da hidratação do cimento. Além disso, a facilidade de aplicação, a consolidação, a durabilidade e a resistência do concreto dependem dessas propriedades [3]. Desde que Powers e Wiler [T. C. Powers, E. M. Wiler, A device for studying the workability of concrete, Proc. Am. Soc. Testing Mater. 41 (1941) 1003-1015.] introduziram seu "plasticímetro" em 1941, diversas tentativas têm sido feitas para se aplicar conceitos fundamentais ao estudo das propriedades do concreto fresco. Um grande avanço foi obtido em 1973, quando Tattersall [G. H. Tattersall, "Relationships between the British standard test for workability and two-point test", Magazine of Concrete Res. 25, 84 (1973) 169-174] introduziu o ensaio de dois pontos da trabalhabilidade e, desde então, a investigação do comportamento reológico do concreto fresco tem sido realizada continuamente [4]. Nos últimos anos, a reologia dos concretos tem sido estudada com determinações que variam entre métodos de ensaio simples e práticos, como o ensaio de abatimento de tronco de cone, até equipamentos mais sofisticados que determinam as curvas de cisalhamento do material, como os reômetros. No entanto, ainda não é muito comum o uso dessa ciência para se estudar as propriedades do concreto fresco e a principal dificuldade está relacionada com as grandes partículas de agregado graúdo que compõe a mistura, as quais impossibilitam o uso de reômetros tradicionais, tais como o viscosímetro capilar ou o viscosímetro cilíndrico. Porém, equipamentos com dimensões suficientemente grandes têm sido construídos, possibilitando a obtenção das curvas de cisalhamento desses materiais.

\section{CLASSIFICAÇÃO REOLÓGICA DO CONCRETO}

Todos os métodos de ensaio normalizados, bem como qualquer outro método empírico, tentam avaliar as propriedades do concreto fresco em termos de uma quantidade única, de maneira que todos eles abrangem a consideração implícita de que esse material se comporta da maneira mais simples possível, como um fluido newtoniano. Porém, uma observação mais casual do seu comportamento mostra que essa suposição não é correta, uma vez que o concreto exige a imposição de uma tensão ou força mínima para o início do escoamento [5], indicando que o seu comportamento não pode ser caracterizado por uma constante única. Assim, para identificar a natureza reológica desse material, é necessário desenvolver alguns experimentos nos quais as medidas são feitas para uma série de velocidades de escoamento distintas.
A maioria das equações usadas para suspensões, como o concreto, tenta relacionar a concentração de uma suspensão com a sua viscosidade ou a tensão de cisalhamento com a taxa de cisalhamento, assumindo, assim, que existe apenas um valor de viscosidade para todo o sistema [1]. As equações que relacionam a concentração com a viscosidade são usadas para descrever o comportamento no escoamento de pastas de cimento, não sendo aplicáveis ao concreto em função da complexidade da suspensão. As equações que relacionam a tensão de cisalhamento e a taxa de cisalhamento, comumente usadas para descrever o comportamento no escoamento do concreto fresco, são apresentadas na Tabela I.

Tabela I - Equações que relacionam a tensão e a taxa de cisalhamento para descrever o comportamento do escoamento de concretos no estado fresco [1].

[Table I - Equations relating stress and shear rate to describe flow behavior of fresh concretes [1].]

\begin{tabular}{cc}
\hline Modelo reológico & Equação do modelo \\
\hline Newton & $\tau=\mu \dot{\gamma}$ \\
Bingham & $\tau=\tau_{0}+\mu \dot{\gamma}$ \\
Herschel-Bulkley & $\tau=\tau_{0}+\mathrm{K} \dot{\gamma}^{\mathrm{n}}$ \\
$\tau=\mathrm{A} \dot{\gamma}^{\mathrm{n}}$ & $n=1$, para fluido \\
& newtoniano \\
Lei das potências & $n>1$, para fluido dilatante \\
& $n<1$, para fluido \\
& pseudoplástico \\
Vom Berg, & $\tau=\tau_{0}+$ Bsen $^{-1}(\dot{\gamma} / \mathrm{C})$ \\
Ostwald-de-Waele & $\tau=\mathrm{a}(\dot{\gamma}+\mathrm{C})^{\mathrm{b}}$ \\
Robertson-Stiff & $\tau=\mathrm{a} \dot{\gamma}+\mathrm{Bsen}^{-1}(\dot{\gamma} / \mathrm{C})$ \\
Eyring & $\dot{\gamma}=\alpha \tau^{2}+\beta \tau+\delta$ \\
Atzeni et al. &
\end{tabular}

Obs.: $\tau=$ tensão de cisalhamento; $\tau_{o}=$ tensão de escoamento; $\mu=$ viscosidade; $\dot{\gamma}=$ taxa de cisalhamento; $A, a, B, b, C, K, \alpha, \beta, \delta=$ constantes.

Os modelos reológicos independentes do tempo - fluidos plásticos (binghamianos), pseudoplásticos e dilatantes permitem ajustar os dados da tensão de cisalhamento, taxa de cisalhamento e viscosidade a tendências específicas. Porém, nenhum desses modelos está isento de erros estatísticos. Normalmente, é difícil computar a tensão de cisalhamento para a taxa de cisalhamento zero (tensão de escoamento). Esse problema pode ser superado pela aplicação de diferentes modelos reológicos capazes de predeterminar estatisticamente a tensão de escoamento. Usando tais modelos, também é possível estabelecer uma viscosidade geral - denominada viscosidade plástica - para uma determinada variação da taxa de cisalhamento. Deve-se compreender que as diferentes equações constitutivas tentam prever as mesmas propriedades e, antes de aceitar um modelo em particular, é importante verificar se as predeterminações do modelo são fisicamente significativas e correspondem ao tipo de comportamento que se está tentando obter [6].

$\mathrm{Na}$ literatura, devido a uma vasta evidência experimental 
das propriedades reológicas do concreto no estado fresco, concluiu-se que o material se comporta como um fluido plástico ou binghamiano $[1,5,7]$ para o intervalo das taxas de cisalhamento envolvidas no uso do material. Assim, a tensão necessária ao escoamento do material - tensão de cisalhamento $(\tau)$ - é igual à soma da tensão de escoamento $\left(\tau_{o}\right)$ e de outro termo proporcional à taxa de cisalhamento $(\dot{\gamma})$, denominada viscosidade plástica $(\mu)$ (equação A).

$$
\tau=\tau_{0}+\mu \dot{\gamma}
$$

Como o concreto fresco é um material heterogêneo, com descontinuidades mecânicas internas extremas, a consideração de um meio contínuo torna-se inaceitável. Assim, os parâmetros reológicos são normalmente medidos como dependentes do equipamento utilizado no ensaio e expressos em unidades de torque, resultando em curvas de cisalhamento onde o torque ( $\mathrm{T}$ ) é apresentado como uma função da velocidade de rotação $(\mathrm{N})$, cuja relação é dada segundo a equação:

$$
\mathrm{T}=\mathrm{g}+\mathrm{h} * \mathrm{~N}
$$

onde $g$, torque de escoamento, em $[\mathrm{Nm}]$, é a intersecção com o eixo do torque e $h$, viscosidade de torque, em [Nm.s], é a inclinação da reta. Essas duas constantes são análogas à tensão de escoamento e a viscosidade plástica, respectivamente [8].

A explicação para o comportamento de um fluido plástico ou binghamiano é que o material, em repouso, apresenta uma estrutura tridimensional com rigidez suficiente para resistir a qualquer tensão inferior à tensão de escoamento e se comporta como um sólido elástico rígido. Caso a tensão de escoamento seja excedida, a estrutura se desintegra e o sistema se comporta como um fluido newtoniano. Quando a tensão de cisalhamento aplicada for reduzida e atingir valores inferiores à tensão de escoamento, a estrutura tridimensional é recuperada [9].

\section{Parâmetros reológicos}

Recentemente, uma metodologia quantitativa fundamental foi desenvolvida para avaliar as propriedades do concreto no estado fresco e esse método introduziu parâmetros reológicos para quantificar a capacidade de escoamento desses materiais. Sabe-se que para uma ampla variação da consistência, o concreto fresco se comporta como um fluido plástico ou binghamiano, sendo que para sua curva de cisalhamento, a intersecção da reta com o eixo tensão de cisalhamento corresponde à tensão de escoamento e a inclinação da reta, à viscosidade plástica. A tensão de escoamento está relacionada com o valor de abatimento do concreto, enquanto a viscosidade plástica é associada às capacidades de aplicação, bombeamento, acabamento e segregação do material e diferencia um concreto facilmente trabalhável de um que apresenta um comportamento "pegajoso", difícil de ser bombeado e com vazios na superfície do elemento estrutural quando a fôrma é retirada $[10,11]$. A tensão de escoamento é uma característica comum do concreto fresco e de materiais granulares secos (como os solos), enquanto a viscosidade plástica tende a relacionar o concreto fresco com outros materiais viscosos (como a água e o óleo). Dessa maneira, pode-se considerar que a tensão de escoamento resulta do atrito intergranular durante o cisalhamento do concreto, enquanto a viscosidade plástica é uma assinatura macroscópica do escoamento da água nos poros de um sistema granular [10]. A interpretação física da tensão de escoamento é que ela corresponde à tensão que precisa ser aplicada no material a fim de que seu escoamento inicie. Quando a tensão de cisalhamento é maior que a tensão de escoamento, a mistura escoa e sua resistência ao escoamento depende da viscosidade plástica [12]. A presença de uma tensão de escoamento nas misturas de concreto não tem sido explicada em termos de microestrutura desse material. Como a concentração de sólidos em um concreto é muito alta e o tamanho e a morfologia desses sólidos podem variar, a tensão de escoamento poderá ter sua origem em três fontes principais [13]: o imbricamento mecânico do agregado graúdo (de forma irregular) que compõe o concreto; as forças de atração entre o cimento e outras pequenas partículas que promovem a floculação; e o gel coloidal de silicato de cálcio hidratado que se forma ao redor das partículas de cimento, como resultado da sua reação de hidratação, bem como outros compostos que contribuem com o endurecimento final das misturas à base de cimento.

A viscosidade plástica é governada pela concentração relativa da mistura, definida como a razão entre a proporção de materiais sólidos (em volume) e sua densidade de empacotamento. A contribuição das várias frações de grãos na viscosidade plástica se dá apenas na extensão em que elas favorecem a densidade de empacotamento da mistura seca correspondente [10]. Diferente de suspensões unimodais, para as quais a viscosidade varia em função da concentração de sólidos, a determinação da viscosidade plástica de uma suspensão de múltiplos componentes é mais complicada. De fato, essas misturas podem ser consideradas como uma suspensão bimodal onde as partículas sólidas estão suspensas em uma solução de pasta. Dessa maneira, a viscosidade de suspensões multimodais está relacionada com a viscosidade da pasta, o volume de sólidos e o grau de aglomeração das partículas - esse último sendo controlado pelo teor de água, presença de superplastificante, forma e textura superficial das partículas sólidas [14].

A relevância em se medir tanto a tensão de escoamento quanto a viscosidade plástica pode ser observada ao comparar os parâmetros reológicos de dois concretos quaisquer (Fig. 1): esses materiais podem ter um dos parâmetros reológicos idênticos, enquanto o outro pode ser totalmente distinto, o que implica no fato desses materiais apresentarem comportamentos reológicos muito diferentes [1].

Assim, a determinação de ambos os parâmetros reológicos permite a diferenciação imediata de concretos que poderiam ser erroneamente considerados idênticos pelos métodos de ensaio padrões existentes. Além disso, os vários fatores que 

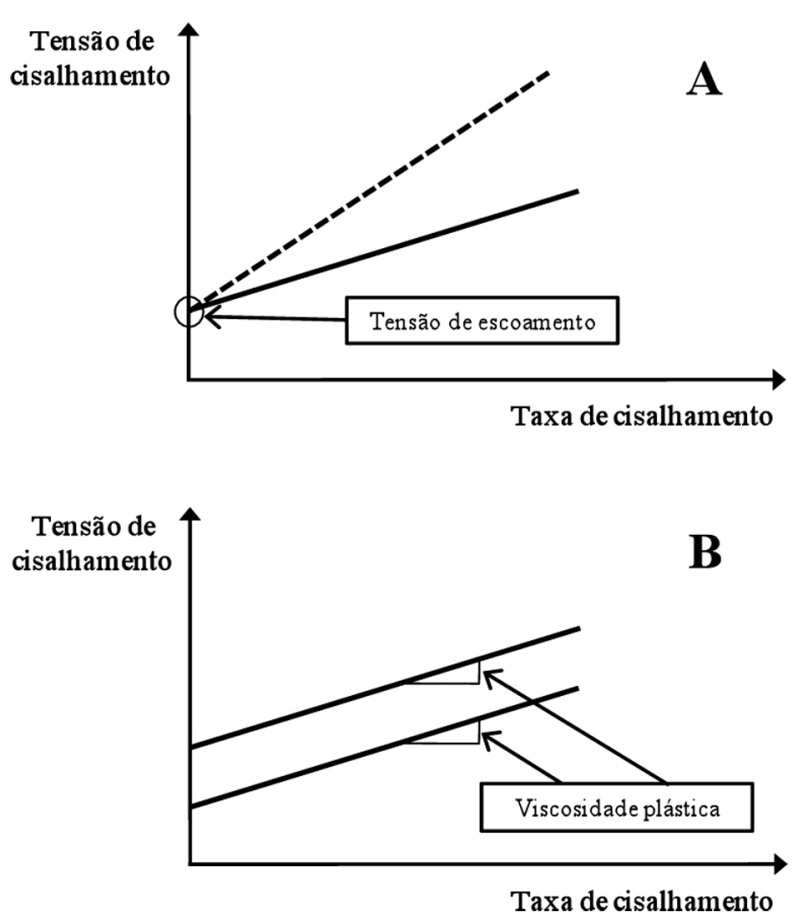

Figura 1: Reologia do concreto: (A) mesma tensão de escoamento e diferentes viscosidades; (B) mesma viscosidade e diferentes tensões de escoamento [1].

[Figure 1: Concrete rheology: (A) same yield stress and different viscosities; (B) same viscosity and different yield stresses [1].]

compõem uma mistura de concreto e que interferem na sua trabalhabilidade influenciam a tensão de escoamento e a viscosidade plástica de maneiras distintas. Sendo assim, o estudo da natureza das mudanças que ocorrem na mistura pode indicar o fator responsável pelas mesmas. A evolução dos parâmetros reológicos está se tornando cada vez mais importante à medida que as novas gerações de concretos se mostram dependentes das condições vigentes durante a concretagem. Mudanças da resistência ao cisalhamento com o tempo, quando um material apresenta um comportamento binghamiano, são conseqüências das mudanças da tensão de escoamento e da viscosidade plástica, as quais normalmente variam exponencialmente com o decorrer do tempo. Do ponto de vista tecnológico, é muito importante saber como a mudança desses parâmetros contribui com as mudanças da resistência ao cisalhamento $[12,15]$. Para os concretos de alto desempenho, um problema comum encontrado na prática das construções é sua rápida perda de trabalhabilidade. Para garantir um comportamento reológico estável por um determinado período de tempo, a caracterização adequada da evolução da trabalhabilidade é importante, o que pode ser facilmente monitorado com o auxílio de um reômetro. Neste equipamento, a amostra de concreto pode ser mantida pelo tempo necessário para que sucessivos ensaios de cisalhamento sejam executados, possibilitando acompanhar a evolução das constantes reológicas. Assim, a evolução da trabalhabilidade de um concreto pode ser descrita pela evolução dos parâmetros reológicos e determinada pelos seus efeitos combinados para uma aplicação em particular. Geralmente, o processo de perda da trabalhabilidade é refletido por um aumento da tensão de escoamento, enquanto que, na maioria dos casos, a viscosidade plástica é praticamente constante durante o período em que o ensaio é realizado (normalmente por menos de $90 \mathrm{~min}$ ) [7]. O aumento da tensão de escoamento ao longo do tempo reflete o processo de endurecimento do concreto; porém, a evolução da viscosidade plástica não. Como as amostras permanecem em repouso entre os consecutivos ensaios reológicos, a exigência de torque para as baixas velocidades de rotação aumenta mais do que para as velocidades mais altas, provavelmente devido à floculação das partículas de aglomerantes, ao crescimento dos produtos da hidratação e ao esgotamento das moléculas de superplastificante no processo químico complexo. Isso resulta em uma redução da inclinação da curva de cisalhamento, o que pode explicar o fato da viscosidade plástica não aumentar com o tempo, tendendo a diminuir ligeiramente em alguns casos [16]. Normalmente o concreto de alto desempenho possui baixa tensão de escoamento e alta viscosidade plástica comparado a um concreto convencional [8]. Uma menor tensão de escoamento significa que esse material está apto a escoar sob seu peso próprio, enquanto uma maior viscosidade plástica é necessária para evitar a segregação dos agregados.

\section{Tixotropia}

O fenômeno da tixotropia é caracterizado pela redução da tensão de cisalhamento ao longo do tempo, enquanto a taxa de cisalhamento é mantida constante. Isto se deve à existência de uma estrutura instável no material, que pode ser rompida com uma determinada energia [7]. Deve ser notado que essa quebra estrutural é reversível, mas raramente para fluidos contendo cimento, uma vez que reações químicas contínuas modificam as propriedades do material com o tempo de maneira irreversível [17]. Para pequenos intervalos de tempo, os processos de aglomeração e de dispersão dominam, resultando nos efeitos tixotrópicos (reversíveis), enquanto que para maiores intervalos de tempo, o processo de hidratação prevalece, resultando em evoluções irreversíveis do comportamento do fluido. De fato, esses dois efeitos podem atuar em qualquer momento, porém eles parecem ter tempos característicos bastante distintos. Como conseqüência, é razoável considerar que existe um período intermediário para o qual os efeitos irreversíveis ainda não se tornaram significantes. Assim, é possível modelar a tixotropia apenas dentro de curtos períodos de tempo, para os quais as evoluções irreversíveis da hidratação podem ser desprezadas, isto é, por períodos de até 30 min após o contato inicial entre o cimento e a água de mistura [18]. Na prática, um concreto é considerado tixotrópico se ele aglomerar muito rapidamente em repouso e se tornar aparentemente cada vez mais fluido durante um cisalhamento com duração de alguns décimos de segundos, ou seja, é um concreto que apresenta um tempo característico de aglomeração bastante curto (normalmente de diversos minutos) e um tempo 
característico de dispersão de alguns centésimos de segundo sob uma taxa de cisalhamento variando entre $1 \mathrm{~s}^{-1}$ e $10 \mathrm{~s}^{-1}$ [18]. Rigorosamente, todos os concretos são tixotrópicos. Como regra geral, os fluidos tixotrópicos se comportam como fluidos plásticos ou binghamianos sob cisalhamento e seu comportamento é definido pela tensão de escoamento e pela viscosidade plástica [17]. Durante os últimos anos, a tixotropia de materiais cimentícios vem sendo quantificada pela medida da área do que é denominado "volta tixotrópica" ou, pelo menos, a área ligada a essa volta. No entanto, este método é muito dependente do equipamento utilizado e do procedimento de ensaio adotado. Ele é baseado no fato de que, devido à natureza transitória da tixotropia e à dependência da resposta reológica na história de cisalhamento, as curvas de cisalhamento medidas sucessivamente em um reômetro durante as seqüências crescentes e decrescentes da taxa de cisalhamento aplicada não serão sobrepostas: durante a parte ascendente da taxa de cisalhamento, a dispersão ocorre, porém não suficientemente rápida para alcançar a tensão de cisalhamento do estado estacionário e a tensão medida é sempre maior do que tensão que seria obtida se o estado estacionário fosse alcançado. Por outro lado, durante a parte descendente da taxa de cisalhamento, a aglomeração ocorre, mas, de novo, não suficientemente rápida para alcançar o estado estacionário e a tensão medida é menor do que a do estado estacionário. Assim, a área entre as duas partes da curva de cisalhamento é medida e considerada como uma representação do trabalho necessário para romper algumas ligações inicialmente presentes em um volume unitário de material. Porém, esse método de medida não fornece um valor intrínseco de qualquer parâmetro reológico físico. Dessa maneira, não existe nenhuma possibilidade além de relações empíricas para a utilização dos resultados obtidos no estudo das conseqüências da tixotropia. Isso parece limitar a maneira de se ter uma classificação reológica dos concretos ou de comparar qualitativamente o efeito dos diversos aditivos. Além disso, nos resultados, a aglomeração e a dispersão não podem ser separadas. A aplicação de uma força vibratória pode anular a tixotropia de um concreto; porém, ainda assim, o comportamento tixotrópico pode ser rapidamente recuperado. No entanto, é possível que o valor de torque medido possa ser maior após um tempo de repouso mais longo, no caso de uma recuperação incompleta da tixotropia do material [7].

\section{FATORES QUE INFLUENCIAM A REOLOGIA DO CONCRETO}

O concreto no estado fresco pode ser considerado um tipo de suspensão concentrada. Nesse caso, o comportamento reológico se afasta do modelo newtoniano e passa a depender de fatores como a concentração volumétrica de sólidos, a característica do meio líquido, a temperatura, as características físicas das partículas e o tipo de interação entre elas. Quando agentes dispersantes são incorporados, o comportamento se torna ainda mais complexo e outros fatores também devem ser considerados - a concentração de molécula de dispersante no meio líquido, o peso molecular, a conformação espacial da molécula de dispersante e a espessura da camada de moléculas de dispersante adsorvidas em tornos das partículas [19, 20]. Assim, dependendo das particularidades de cada suspensão e da magnitude da taxa de cisalhamento aplicada, um ou mais fatores pode prevalecer e comandar o comportamento reológico do fluido.

\section{Materiais constituintes da mistura}

As propriedades reológicas do concreto fresco variam amplamente de acordo com os materiais constituintes da mistura e com as condições de produção e do meio ambiente no qual o material está inserido. A reologia dos concretos de alto desempenho pode ser influenciada por diversos parâmetros relacionados com o cimento, o superplastificante e/ou a interação entre eles, isto é, com a composição química e fases componentes do cimento (especialmente os teores de $\mathrm{C}_{3} \mathrm{~A}$ e álcalis), a granulometria do cimento, a quantidade e o tipo de sulfato de cálcio presente no cimento, a natureza química e peso molecular do superplastificante, o grau de sulfonatação e a natureza dos íons contrários do superplastificante, a dosagem e o método de adição do aditivo [12, 21-23]. Além disso, a composição, a morfologia e a distribuição granulométrica dos agregados, a dosagem do concreto (especialmente a relação água/cimento), a temperatura, a seqüência e a intensidade de mistura também podem influenciar as propriedades reológicas desses materiais. Dependendo da dosagem de um concreto, um aumento no teor da água de mistura pode resultar em um aumento de suas propriedades reológicas, com uma redução uniforme tanto da tensão de escoamento quanto da viscosidade plástica. Para um determinado conjunto de materiais, o teor de água pode ser um parâmetro para o controle da viscosidade do material [24], enquanto a tensão de escoamento está diretamente relacionada com o seu abatimento.

A influência dos agregados sobre as propriedades reológicas do concreto fresco está relacionada principalmente com a quantidade de agregado que compõe a mistura e com a sua granulometria: os parâmetros reológicos aumentam com o teor de agregado, porém ambos são reduzidos quando a distribuição granulométrica do agregado se torna mais ampla [25]. Devido às altas taxas de reação da fase aluminato, elas são muito importantes quando da determinação das características reológicas dos materiais à base de cimento nos primeiros estágios da hidratação. Assim, no caso do cimento, a influência mais significativa está relacionada com sua composição química, isto é, com o retardamento do processo de hidratação dos aluminatos causados pelo sulfato de cálcio. Normalmente, o efeito dos produtos de hidratação sobre a tensão de escoamento é mais evidente do que sobre a viscosidade plástica, sendo essa influência maior à medida que a disponibilidade de sulfatos para a formação dos cristais de etringita diminui [26].

Em termos de reologia, os superplastificantes são considerados interferências positivas em uma mistura 
de concreto fresco. Eles são utilizados para aumentar a trabalhabilidade desse material de tal forma que se obtenham misturas fluidas, com tensão de escoamento tão reduzida que pouca ou nenhuma vibração se torna necessária. Embora existam algumas exceções, a adição de superplastificante às misturas à base de cimento possui duas consequiências principais: uma redução significativa da tensão de escoamento e um pequeno ou nenhum efeito sobre a viscosidade plástica, atribuídas à defloculação das partículas de cimento [13, 27]. Assim, com a utilização de superplastificantes, é possível controlar a reologia do concreto sem alterar a relação água/ cimento, o que influencia diretamente a durabilidade e a resistência do material [28].

Do ponto de vista da reologia, a adição mineral produz efeitos intensos sobre as propriedades do concreto ao qual é incorporada, tal como o aumento da área superficial. Como a adição mineral usualmente possui partículas muito pequenas, suas partículas são muito reativas quimicamente e adsorvem moléculas de superplastificante $[11,29]$. Como resultado, a tensão de escoamento e a viscosidade plástica tendem a aumentar. Porém, a substituição de parte do cimento por uma adição mineral também pode resultar em uma redução de ambos os parâmetros reológicos do concreto. A melhor distribuição granulométrica do aglomerante e o efeito lubrificante imposto pelas pequenas partículas da adição mineral - possivelmente diminuindo o imbricamento entre os agregados - estão relacionados com a redução dos parâmetros reológicos e, quanto menor e mais esféricas forem as partículas das adições, maior a redução observada $[16,30]$.

\section{Seqüência e intensidade de mistura}

$\mathrm{O}$ processo de mistura de um material à base de cimento envolve a quebra de aglomerados de partículas. Em um material bem misturado, a quantidade de aglomerados em suspensão é pequena e as partículas estão dispersas; com isso, existem poucas ligações entre as membranas dos hidratos susceptíveis à ruptura pela aplicação de um cisalhamento adicional [31]. Além disso, o grau de uniformidade de um lote de concreto também depende da sequiência de incorporação dos vários materiais constituintes da mistura [5, 27], fazendo com que as propriedades reológicas dos materiais à base de cimento sejam sensíveis à seqüência e intensidade de mistura. A taxa de cisalhamento aplicada durante a mistura pode variar e, assim, constituir uma das variáveis que mais interferem nas propriedades reológicas dos materiais à base de cimento: o aumento da taxa de cisalhamento reduz a viscosidade plástica desses materiais e diminui a área de histerese formada entre as partes ascendente e descendente da curva de cisalhamento. Estudos realizados indicam que uma estrutura aglomerada pode ser quebrada à medida que a taxa de cisalhamento é aumentada; porém, para taxas de cisalhamento muito altas, os produtos da hidratação são liberados na fase aquosa resultando em uma redução das propriedades no escoamento [31]. Além disso, como a energia disponível para a mistura diminui desde a produção do concreto (misturador da usina) até o local da construção (caminhão-betoneira), a aglomeração do material aumenta e sua fluidez diminui [18]. Assim, o processo de produção de uma mistura é muito importante, pois a história do cisalhamento influencia as propriedades reológicas dos materiais à base de cimento no estado fresco [32].

\section{Temperatura}

Apesar da reologia de fluidos plásticos ou binghamianos não ser muito sensível à temperatura, a taxa de mudança do comportamento reológico com o tempo de um sistema quimicamente reativo contendo cimento é influenciada por esse fator [33]. Assim, o mesmo deve ser considerado. As mudanças na temperatura podem influenciar a reologia de materiais à base de cimento por meio de vários mecanismos. A temperatura pode ter um efeito marcante sobre a taxa de adsorção do superplastificante sobre os grãos de cimento, o que, em termos, é influenciado pela taxa de hidratação do cimento e pela concentração de íons sulfato presente na solução aquosa. Dessa maneira, dependendo da temperatura da mistura, parece existir uma competição entre a adsorção do aditivo e a concentração de íons sulfato na solução: o primeiro pode promover a dispersão das partículas de cimento e prolongar a manutenção da fluidez, enquanto o último pode contribuir com a diminuição da intensidade do efeito estérico do polímero, resultando em um aumento exponencial da tensão de escoamento da mistura [20]. O tipo de aditivo usado com um determinado cimento normalmente resulta em diferentes propriedades reológicas dependendo da temperatura do concreto [12]. Sob altas temperaturas, o concreto fresco endurece em um intervalo de tempo menor quando comparado com as condições normais e essa pega rápida diminui a trabalhabilidade durante os processos de aplicação, adensamento e acabamento do material. De uma forma geral, os tempos de início e fim de pega diminuem à medida que a temperatura de cura aumenta, o que obviamente está relacionado com o aumento da taxa de hidratação do cimento com a temperatura, principalmente nos primeiros instantes [3]. Uma queda na temperatura normalmente diminui o valor da tensão de escoamento, mas aumenta substancialmente o valor da viscosidade plástica, enquanto um aumento na temperatura faz com que esses parâmetros se modifiquem na direção oposta. A variação da mudança dos valores de ambos os parâmetros reológicos devido à temperatura também depende da relação água/cimento e da dosagem de superplastificante. Assim, a mudança na resistência ao cisalhamento dos materiais à base de cimento pode ser qualitativamente diferente para diferentes relações água/cimento e dosagens de superplastificante [12]. Para misturas de composições diferentes incorporadas com um mesmo tipo de superplastificante, as mudanças da tensão de escoamento ao longo do tempo variaram em função da temperatura da mistura. Abaixo de uma determinada temperatura, as misturas exibem uma considerável manutenção (ou até mesmo redução) da tensão de escoamento durante cerca de $30 \%$ do período de indução 
da hidratação, enquanto um grande aumento no valor dessa tensão é observado no período seguinte. Acima da temperatura limite, as misturas apresentam um aumento linear da tensão de escoamento ao longo do tempo [20].

\section{Reologia da pasta de cimento}

De um ponto de vista prático, o concreto fresco pode ser considerado como um material bifásico, composto por uma fase matriz (pasta de cimento) e uma fase partícula (agregados). Assim, seu comportamento no escoamento pode ser considerado dependente principalmente da viscosidade da matriz e da porcentagem volumétrica de agregado [8]. Como a pasta de cimento envolve os agregados em um concreto, ao manter constante a dimensão máxima característica e a quantidade de agregados presentes na mistura, as propriedades reológicas do concreto podem ser relacionadas com aquelas da pasta de cimento [34]. Como a pasta de cimento corresponde à maior parte da área superficial do concreto e é a responsável pela fluidez e pela coesão do concreto, a trabalhabilidade e demais propriedades reológicas desse material são dependentes de suas características [35]. As propriedades reológicas do concreto e da pasta de cimento no estado fresco são complexas em diferentes maneiras e medidas em pastas nem sempre predizem as determinações nos concretos correspondentes. Existe uma percepção geral de que estabelecer uma conexão entre a reologia de um concreto e a reologia de uma pasta de cimento está muito além de nossa capacidade [5]. A principal razão para isso é que a reologia da pasta de cimento é medida sob condições nunca experimentadas pela mesma no concreto. Com isso, os valores dos parâmetros reológicos medidos para a pasta de cimento podem diferir daqueles obtidos para os parâmetros do concreto: os valores usualmente relatados na literatura para as pastas de cimento não consideram a participação dos agregados, que agem como redutores de calor e cisalham a pasta de cimento durante o processo de mistura do concreto [32]. Ainda assim, o conhecimento das propriedades reológicas da pasta de cimento pode fornecer informações importantes sobre o comportamento reológico dos concretos. Se medidas corretamente, as propriedades no escoamento da pasta de cimento podem ser usadas para controlar as adições químicas e minerais incorporadas à mistura de concreto. $\mathrm{O}$ comportamento reológico das pastas de cimento, em função do tempo e da dosagem de superplastificante, fornece informações relevantes sobre algumas propriedades chave - tais como o abatimento - que podem ser transferidas ao concreto. Dessa maneira, a compatibilidade de um determinado par cimentosuperplastificante pode ser inicialmente estudada por meio da determinação da fluidez de pastas de cimento pelo ensaio de miniabatimento e cone de Marsh, cujos resultados obtidos podem ser posteriormente validados nos concretos [21].

As novas aplicações das pastas de cimento, como o concreto de alto desempenho, necessitam de um melhor controle de suas propriedades reológicas e do fenômeno da segregação. A tendência geral leva à formulação de pastas muito concentradas, apesar de bastante fluidas, com baixas tensões de escoamento, mediante o controle de sua composição química - especialmente com relação aos teores de aluminatos e de sulfatos do cimento - distribuição granulométrica dos concretos e, finalmente, das interações interpartículas pela adsorção do superplastificante [36]. Simultaneamente, a homogeneidade da mistura deve ser mantida a fim de se evitar a segregação.

A reologia e estabilidade de pastas de cimento muito concentradas, em um regime onde o volume de líquidos está próximo do necessário para preencher os vazios do empacotamento das partículas, podem se aproximar tanto de conceitos desenvolvidos para a reologia de suspensões extrapoladas para uma fração de empacotamento máximo quanto de conceitos físicos e mecânicos de materiais granulares [36]. De uma maneira geral, o comportamento reológico das pastas de cimento fresco é semelhante ao dos concretos, ou seja, se aproxima de um fluido plástico ou binghamiano. Porém, por se tratar de um sistema complexo, as pastas de cimento podem apresentar outros comportamentos reológicos dependendo das condições de ensaio, tais como a composição da pasta, o estado de dispersão das partículas e o histórico de cisalhamento. O papel desempenhado pelas condições experimentais - tais como o tempo e a intensidade de mistura, a duração do ciclo de cisalhamento, o número de repetições e a geometria do sistema de medida - na determinação dos parâmetros reológicos da pasta de cimento mostra que diferenças freqüentemente grosseiras e, alguns casos, até contraditórias podem surgir nos resultados obtidos. Conseqüentemente, qualquer contribuição para a compreensão da reologia das pastas de cimento deve seguir necessariamente condições de ensaio padronizadas.

\section{Dispersão e empacotamento de partículas}

As partículas de cimento apresentam uma grande tendência à aglomeração quando entram em contato com um líquido tão polar como a água. Essa aglomeração, permanente ou não, pode interferir no empacotamento e, conseqüentemente, na reologia das suspensões. Assim, a eliminação dos aglomerados de partículas resulta em uma série de vantagens em termos de propriedades reológicas das suspensões. Para se conseguir uma distribuição homogênea da água e um ótimo contato água/cimento, as partículas de cimento deverão estar corretamente afastadas e conservadas em um alto estado de dispersão. As suspensões dispersas, além de apresentarem menor viscosidade, permitem a utilização de maiores concentrações de sólidos. Nelas, as partículas encontram-se individualizadas e, por isso, são pouco influenciadas pela força da gravidade [19]. Dessa maneira, a homogeneidade e a estabilidade das suspensões são mantidas por um intervalo de tempo maior. Para se obter suspensões dispersas, as forças de repulsão entre as partículas devem exceder as forças de atração. Assim, o uso de dispersantes torna-se necessário: esses aditivos são eficientes na quebra das estruturas de partículas de cimento e em sua dispersão, evitando uma ligação prematura, 
minimizando a quantidade de água necessária para a adequada fluidez da suspensão e resultando em misturas mais trabalháveis pelo intervalo de tempo desejado.

As condições em uma suspensão à base de cimento podem ser modificadas a fim de se evitar a formação de uma estrutura aglomerada. Os três mecanismos básicos de dispersão são [19]: eletrostático, que corresponde ao desenvolvimento de cargas elétricas na superfície das partículas em decorrência da sua interação com o meio líquido; estérico, que corresponde à adsorção superficial de polímeros de cadeias longas que dificultam a aproximação das partículas por impedimento mecânico; e eletroestérico, que corresponde à adsorção específica de moléculas com grupos ionizáveis ou polieletrólitos na superfície das partículas, no qual os íons provenientes da dissociação desses grupos ionizáveis somam uma barreira eletrostática ao efeito estérico. Além desses, outros mecanismos também podem contribuir para a fluidificação da mistura, tais como: redução da tensão de superfície da água; filme lubrificante entre as partículas de cimento; dispersão dos grãos de cimento, liberando a água aprisionada entre os aglomerados; inibição da reação de hidratação na superfície das partículas de cimento, deixando mais água livre para fluidificar a mistura; mudança na morfologia dos produtos de hidratação [37, 38].

A fluidez inicial de soluções fortemente suspensas, tais como a pasta de cimento e o concreto, também depende de considerações físicas, isto é, da distribuição granulométrica, do índice de forma e da textura superficial das partículas [39]. Sendo assim, o projeto da distribuição de tamanho de partículas é de fundamental importância, pois promove o empacotamento e, juntamente com o fluido, define as características reológicas do material durante o processo de mistura e quando no estado fresco.

Uma série de fatores pode alterar o empacotamento de um determinado conjunto de partículas. O primeiro a ser considerado é a existência de diversas distribuições granulométricas alterando a condição de dispersão inicial. O outro fator que pode alterar a condição de empacotamento é a morfologia das partículas: quanto menos esférica for a partícula, menor será a densidade de empacotamento de uma distribuição que a contenha e, quanto menor o tamanho das partículas irregulares, maior será o efeito do atrito entre elas, devido à maior área superficial específica [19]. A porosidade interna das partículas também altera a densidade de um material. As partículas podem ser totalmente densas, com porosidade interna fechada ou com porosidade aberta. Para efeito de distribuição granulométrica, as partículas que apresentam porosidade fechada são semelhantes às densas, mas resultam em misturas com densidade menor. Já as partículas que apresentam porosidade aberta perturbam o empacotamento devido ao seu formato irregular e também resultam em misturas com menores densidades de empacotamento [19]. A granulometria adequada do aglomerante e o efeito lubrificante imposto pelas partículas finas possivelmente reduzem o imbricamento entre os agregados e, conseqüentemente, a resistência ao escoamento: quanto mais fino e mais esférico for o material cimentício, maior a redução da resistência ao escoamento. Assim, uma determinada granulometria que garanta o empacotamento ótimo das partículas de aglomerantes pode melhorar o comportamento no escoamento do concreto [16].

\section{Vibração}

Uma observação inicial do comportamento do concreto fresco sugere que o material possui uma tensão de escoamento e que a aplicação de uma força vibratória reduz essa tensão consideravelmente, pelo menos, em uma extensão tal que o material possa escoar sob a influência do seu peso próprio [27].

Quando o concreto fresco é submetido à vibração, observam-se mudanças significativas em suas propriedades reológicas. Existe um indício de que a curva de cisalhamento do concreto vibrado passa pela origem, isto é, que o material efetivamente não possui mais uma tensão de escoamento, no entanto, sua curva não é mais uma relação linear simples [5]. Sob vibração, pode-se dizer que o concreto fresco se comporta como um fluido pseudoplástico e possui tensão de escoamento igual à zero, desde que certas condições limites de vibração não sejam excedidas [40]. A vibração tem um efeito considerável sobre a tensão de escoamento dos concretos, sendo esse efeito imediato e também instantaneamente reversível [41]. Porém, as causas da redução da tensão de escoamento devido à vibração ainda não são totalmente compreendidas: especula-se que a vibração dispersa os grãos de cimento e também rompe as fracas ligações químicas iniciais resultantes da gelificação da pasta de cimento fresco, além de fazer com que o agregado graúdo se agite, destruindo o esqueleto inicialmente conectado. Dessa maneira, a redução da tensão de escoamento do concreto submetido à vibração pode estar relacionada com o enfraquecimento das ligações físicas e químicas entre os materiais constituintes da mistura [13].

Com relação à viscosidade plástica, esse parâmetro reológico normalmente muda com a aplicação da vibração e o material se torna pseudoplástico [42, 43]; porém, em algumas situações, ela pode ser pouco influenciada e até aumentar para concretos pouco viscosos [7]. Portanto, mesmo que a viscosidade plástica de uma mistura não seja modificada sob vibração, o concreto se tornará ainda mais trabalhável devido à redução da sua tensão de escoamento.

\section{AVALIAÇÃO DAS PROPRIEDADES REOLÓGICAS DO CONCRETO}

Quando as propriedades reológicas são determinadas em laboratório, diz-se que está se realizando ensaios de reometria. Para isso, existem diversas técnicas experimentais disponíveis que variam de acordo com o material a ser ensaiado. Diversos dispositivos reológicos têm sido introduzidos durante as últimas décadas como uma tentativa de medir corretamente o comportamento no escoamento de uma mistura no estado fresco. Porém, todos 
esses equipamentos operam com princípios de reometria rotacional, o que predetermina uma construção mecânica complicada e um preço relativamente alto. Ao longo dos anos, outros modelos também têm sido desenvolvidos para a dosagem de misturas de concreto por meio de uma aproximação reológica, os quais normalmente não fornecem diretamente um parâmetro reológico mensurável na expressão de dosagem [44].

No caso particular do concreto fresco, os métodos de ensaio utilizados para a determinação de suas propriedades reológicas são divididos em dois grupos, de acordo com os resultados obtidos: um ou dois parâmetros reológicos. Os ensaios mais comuns medem apenas um parâmetro reológico e a relação entre o parâmetro medido e o segundo normalmente é complexa; na maioria das vezes, é impossível calcular o parâmetro fundamental a partir do resultado obtido, podendo apenas garantir uma correlação entre eles.

Para o concreto de alto desempenho, suas propriedades reológicas são avaliadas por meio dos mesmos métodos de ensaio usados na avaliação de um concreto convencional, porém suas características específicas impedem uma correta interpretação dos resultados. Como exemplo, citase o ensaio de abatimento de tronco de cone amplamente utilizado na prática das construções. O ensaio é válido para concretos com abatimento variando entre $25 \mathrm{~mm}$ e $175 \mathrm{~mm}$, o que corresponde a consistências variando entre os estágios de baixa e média plasticidade; como um concreto de alto desempenho normalmente apresenta abatimento maior que $200 \mathrm{~mm}$, esse método de ensaio não parece caracterizar corretamente seu comportamento no estado fresco [8]. Além disso, sabe-se que, na prática, dois concretos com um mesmo valor de abatimento podem apresentar comportamentos reológicos diferentes. A partir desse ensaio pode-se demonstrar que a tensão de escoamento está dentro da variação desejada, mas a viscosidade plástica, que não é medida pelo ensaio, pode ser tão alta que a mistura é rotulada como "pegajosa" e considerada difícil de ser aplicada mesmo sob vibração [1].

Portanto, a avaliação das propriedades reológicas do concreto fresco baseada apenas nos resultados obtidos pelo ensaio de abatimento de tronco de cone é extremamente difícil, se não impossível. A necessidade pela descrição das propriedades reológicas em termos de quantidades físicas fundamentais tem sido amplamente discutida e qualquer ensaio que descreva o comportamento no escoamento do concreto fresco deve avaliar, pelo menos, os dois parâmetros reológicos - tensão de escoamento e viscosidade plástica. Infelizmente, a maioria dos métodos de ensaio existentes mede apenas um desses parâmetros, o relacionado com a tensão de escoamento ou com a viscosidade plástica do material. De fato, os métodos de ensaio que medem apenas um dos parâmetros reológicos podem apenas refletir a resposta do concreto fresco para uma taxa de cisalhamento em particular. Como as suspensões de partículas exibem quebra estrutural ou reconstrução dos aglomerados quando submetidas à uma taxa de cisalhamento variável [45], para se obter uma caracterização mais pertinente do seu comportamento no escoamento, é necessário avaliar as propriedades para uma certa variação da taxa de cisalhamento enquanto o concreto escoa [8].

Atualmente, existem ensaios capazes de fornecer os dois parâmetros reológicos que caracterizam o comportamento de um concreto no estado fresco, porém eles não necessariamente permitem um cálculo direto da viscosidade plástica nem da tensão de escoamento. Quando a dimensão máxima de uma partícula é pequena, como no caso das pastas de cimento, a curva de cisalhamento completa pode ser obtida em um reômetro com um espaçamento de poucos milímetros entre as superfícies cisalhantes e os parâmetros reológicos podem ser determinados através de análises convencionais considerando o escoamento laminar [46]. No caso dos concretos, a avaliação reológica tem encontrado alguns problemas: a dificuldade em desenvolver ensaios e/ ou equipamentos capazes de medir diretamente os valores corretos dos parâmetros reológicos está relacionada à dimensão máxima característica do agregado graúdo, à tendência à segregação e aos efeitos do tempo; porém, algumas soluções alternativas têm sido utilizadas com grande sucesso.

As interações complexas entre a composição da mistura, as características do equipamento de ensaio e o modelo usado na estimativa das propriedades reológicas de materiais à base de cimento impõem um desafio na comparação entre os resultados reológicos obtidos a partir de diferentes fontes. Adicionado ao fato de que o comportamento de materiais à base de cimento é dependente do tempo, da temperatura e da história de cisalhamento, existe a necessidade do desenvolvimento de normas detalhadas e amplas para o ensaio da reologia desses materiais a fim de fazer um estudo criterioso dos diversos resultados reológicos disponíveis [6].

\section{Reômetro}

Reômetros são equipamentos direcionados à avaliação das propriedades reológicas de fluidos e suspensões. Eles permitem estudar o comportamento da tensão de escoamento e da viscosidade plástica em função de outras variáveis, como tempo, temperatura etc. Além disso, os reômetros apresentam geometrias que são adequadas para a avaliação de formulações durante a mistura e o transporte, simulando condições de escoamento turbulento. Dessa maneira, os reômetros são equipamentos precisos não apenas para pesquisa, mas também para estudos práticos e medidas de controle de qualidade do material. Eles fornecem muito mais informações dos que os ensaios empíricos convencionais, reduzindo os custos com material e mão-de-obra. Além disso, as informações obtidas são mais objetivas, uma vez que o ensaio é totalmente automatizado e controlado por computador [47].

O primeiro reômetro desenvolvido especificamente para a caracterização de concretos foi concebido por Powers, em 1968, no conceito de cilindros coaxiais (Fig. 2a). Com a evolução tecnológica dos reômetros, surgiram equipamentos que utilizam novos conceitos para o cisalhamento do material, 

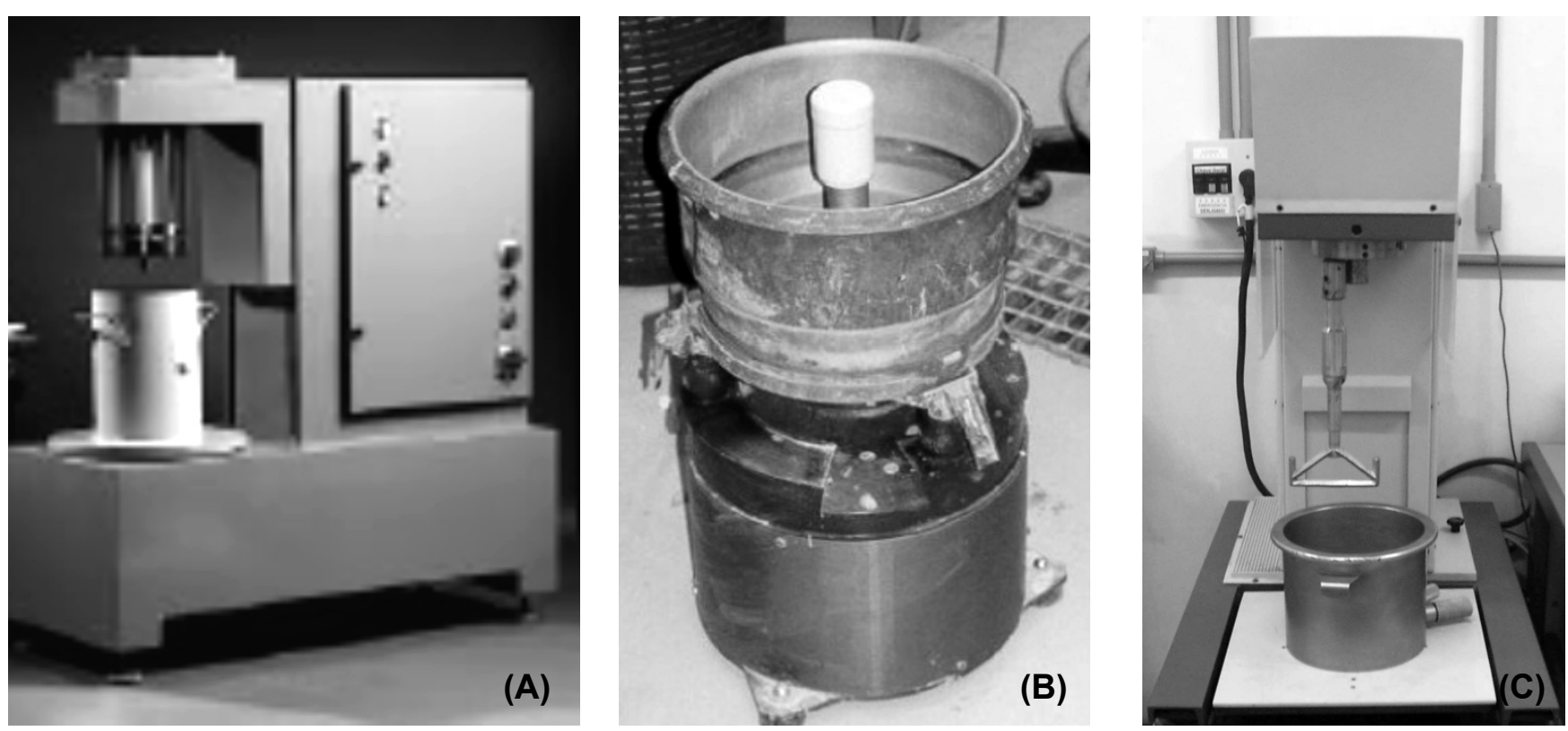

Figura 2: (A) Reômetro BML, baseado no conceito de cilindros coaxiais [50]; (B) Reômetro BTRHEOM, baseado no conceito placa/placa [50]; (C) Reômetro baseado no modelo planetário.

[Figure 2: (A) BML rheometer, based on coaxial cylinders concept [50]; (B) BTRHEOM rheometer, based on platelplate concept [50]; (C) rheometer based on planetary concept.]

como o reômetro BTRHEOM (Fig. 2b) desenvolvido no Laboratoire Central des Ponts et Chaussées (LCPC), França [48], baseado no conceito placa/placa, no qual um anel de concreto de eixo vertical é cisalhado, sendo sua seção inferior fixa e sua seção superior rotativa em torno do eixo. No Brasil, um reômetro destinado à avaliação reológica de concretos refratários (Fig. 2c) foi desenvolvido pelo Grupo de Engenharia de Microestrutura de Materiais (GEMM/ UFSCar). O projeto é baseado em um misturador planetário, o que permite utilizá-lo na avaliação das propriedades reológicas tanto de concretos auto-adensáveis quanto em misturas com fluidez reduzida, o que possibilita ampliar o campo de utilização do equipamento quando comparado aos reômetros de cilindros coaxiais, além de permitir o estudo da mistura e a simulação de diversas técnicas de aplicação dos concretos, dentre outras coisas [49].

Os diversos reômetros disponíveis comercialmente se baseiam em apenas dois princípios básicos de funcionamento [49]: reômetro em que o torque aplicado ao fluido é controlado, sendo avaliado o cisalhamento resultante indicado para avaliações em que a tensão de escoamento é a principal propriedade a ser determinada; e reômetro em que o cisalhamento aplicado ao material é controlado, sendo registrada a força necessária para tal - indicado para avaliações do comportamento da viscosidade do material sob diversas taxas de cisalhamento.

As medidas obtidas a partir de um reômetro só podem ser interpretadas caso o fluido avaliado seja homogêneo. Devido à extensa variação da distribuição granulométrica das partículas presentes em uma mistura de concreto, esse material não pode ser considerado como tal. Assim, a descrição das características dessa classe de material em termos de tensão de cisalhamento e taxa de cisalhamento é imprecisa. Uma melhor aproximação é feita pela análise direta das forças (torque) que resultam do cisalhamento (velocidade de rotação) do concreto [51].

\section{Curvas de cisalhamento}

O comportamento reológico de um material depende das condições de ensaio, tais como a taxa de cisalhamento, a temperatura, a energia de mistura etc. Assim, a avaliação das propriedades reológicas de concretos de alto desempenho necessita de considerações especiais: eles demandam métodos de ensaio que envolvem a avaliação da energia na mistura e, então, o quanto o material se deforma [52]. Normalmente, as propriedades reológicas do concreto fresco são determinadas a partir de curvas de cisalhamento obtidas com o auxílio de um reômetro. Dessa maneira, é possível determinar a relação entre a tensão de cisalhamento e a taxa de cisalhamento sob condições definidas fisicamente. As curvas de cisalhamento são necessárias para caracterizar adequadamente os materiais à base de cimento como fluidos não-newtonianos, uma vez que sua viscosidade depende tanto da taxa de cisalhamento quanto da duração do cisalhamento [53]. Assim, a condição experimental (ciclo de cisalhamento) deve ser definida antes do cálculo dos parâmetros reológicos. Embora não exista nenhuma restrição quanto à seleção da condição de cisalhamento, condições mais simples são freqüentemente adotadas. Para materiais à base de cimento, onde certos fenômenos dependentes do tempo também estão envolvidos, a mudança na velocidade de rotação (taxa de cisalhamento) deve ser feita em escada e não continuamente [54]. Quando um concreto é submetido a uma taxa de cisalhamento gradualmente crescente e, posteriormente, essa taxa é uniformemente reduzida à zero, a 


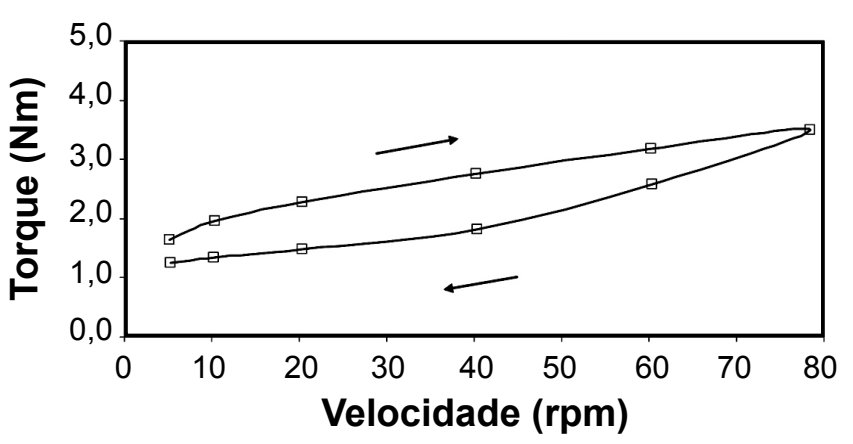

Figura 3: Curva de cisalhamento de um concreto de alto desempenho [55]. Obs.: As setas indicam o sentido de aplicação da velocidade de cisalhamento.

[Figure 3: Flow curve of a high performance concrete [55]. Obs.: the arrows indicate the up and down curves, respectively.]

curva resultante (parte descendente) poderá ou não coincidir com a curva anterior (parte ascendente). A esse gráfico dá-se o nome de curva de histerese, construída quando o material sofre uma quebra estrutural sob a ação de um cisalhamento (Fig. 3) [5]. Esse volume de histerese, cuja área representa o trabalho relacionado com o volume de material cisalhado, permite uma análise qualitativa do cisalhamento, sendo possível, então, tirar conclusões quanto à estabilidade da estrutura desses materiais [53].

A forma das curvas de histerese varia dependendo do tempo total do ciclo de cisalhamento, da taxa de acréscimo e de redução da taxa de cisalhamento e do "ponto de virada" dos ensaios (momento em que a taxa de cisalhamento crescente começa a diminuir). Dentre os parâmetros que promovem variações na forma das curvas de cisalhamento das suspensões, a taxa de coagulação das partículas é o único fator relacionado com sua composição química. Como os outros parâmetros não estão relacionados com a composição e são simples condições experimentais, é importante observar que a variação da forma das curvas de cisalhamento nem sempre descrevem diferenças quanto à qualidade da amostra ensaiada [4, 56]. A variação dos resultados reológicos devido à diferença no atrito de superfície do equipamento de ensaio tem sido atribuída aos efeitos de escorregamento. Nas paredes do equipamento de ensaio, uma redução da densidade da suspensão adjacente à parede sólida ocorre devido à dificuldade geométrica em organizar as partículas e à migração da partícula de regiões de alta taxa de cisalhamento, um fenômeno denominado efeito de parede. Além disso, se o material da parede não tiver afinidade com as partículas dispersas, o meio dispersante tende a formar uma camada que atua como lubrificante, de maneira que existe o escorregamento da suspensão de sólidos ao longo da parede, especialmente sob baixas taxas de cisalhamento. Em tal caso, é desejável uma parede com superfície rugosa, onde a extensão da rugosidade depende da natureza e do tamanho das partículas dispersas [6]. Outra variável que pode influenciar os resultados reológicos é a sedimentação de partículas durante o ensaio reológico, a qual é função da viscosidade e densidade do líquido, diâmetro, forma e densidade da partícula e da concentração volumétrica de partículas dispersas. A sedimentação pode resultar em um aumento da viscosidade, porém, na maioria dos experimentos reológicos, leva a uma redução do torque com o tempo e à interpretação equivocada dos resultados [6].

As curvas de histerese são úteis como um indicador preliminar do comportamento, porém elas não fornecem uma boa base para a análise quantitativa desse comportamento. Uma técnica alternativa é estudar a taxa de quebra estrutural com o tempo sob uma taxa de cisalhamento constante ou, mais precisamente, sob uma velocidade constante.

\section{TENDÊNCIAS PARA O FUTURO}

Tradicionalmente, o comportamento do concreto fresco é relacionado com sua trabalhabilidade, que corresponde a uma propriedade intrínseca do material, sendo relacionada ao tipo de construção e aos métodos de aplicação, adensamento e acabamento do material. Na tecnologia dos concretos, esse termo tem sido freqüentemente usado para abranger todas as qualidades necessárias a uma mistura, porém muita confusão tem sido feita pelo uso descuidado e impreciso da terminologia. A menos que e até que seja possível definir a trabalhabilidade em termos de constantes físicas fundamentais, o termo deve ser restrito ao uso mais geral, sem qualquer quantificação.

No entanto, a simples consideração de que a trabalhabilidade seja o único parâmetro importante deve ser visto com cautela. As dificuldades passam pelas considerações que envolvem os próprios materiais já na fabricação dos concretos - grande variabilidade de matéria-prima, disponibilidade e forma de estocagem desses materiais - até a correção de suas partes na escala do processo industrial, nos equipamentos de mistura, nas condições de transporte, redosagem, aplicação e lançamento do material. Dessa forma, entende-se a trabalhabilidade final como uma suplantação de problemáticas envolvidas até o momento de aplicação do concreto.

Nos últimos anos, a demanda pela estimativa da trabalhabilidade do concreto fresco em termos de constantes reológicas no lugar dos valores obtidos a partir de ensaios empíricos vem aumentando bastante. A caracterização reológica do concreto fresco tem muitas aplicações que podem interessar na estimativa de características como a energia de mistura, a capacidade de bombeamento, a velocidade de escoamento sob o efeito da gravidade e/ou da vibração, o intervalo de tempo durante o qual o concreto pode ser aplicado etc. Assim, verifica-se que a aplicação de conceitos reológicos na avaliação das propriedades dos concretos é de extrema importância para a correta utilização desses materiais.

\section{AGRADECIMENTOS}

Ao $\mathrm{CNPq}$ pela bolsa de estudo concedida e às empresas Holcim Brasil S. A., Elkem Materials South America Ltda., Basf Construction Chemicals Brasil e Mineração Jundu Ltda., pela doação dos materiais utilizados na pesquisa. 


\section{REFERÊNCIAS}

[1] C. F. Ferraris, "Measurement of rheological properties of high performance concrete: state of the art report", J. Res. Nat. Inst. Stand. Technol. 104, 5 (1999) 461-478.

[2] J. E. Wallevik, "Relationship between the Bingham parameters and slump", Cement Concrete Res. 36, 7 (2006) 1214-1221.

[3] M. Heikal, M. S. Morsy, I. Aiad, "Effect of treatment temperature on the early hydration characteristics of superplasticized silica fume blended cement pastes", Cement Concrete Res. 35, 4 (2005) 680-687.

[4] O. H. Wallevik, O. E. Gjørv, "Modification of the twopoint workability apparatus", Magazine Concrete Res. 42, 152 (1990) 135-142.

[5] G. H. Tattersall, P. F. G. Banfill, "The rheology of fresh concrete", Pitman, London, Inglaterra (1983) 347p.

[6] M. Nehdi, M.-A. Rahman, "Estimating rheological properties of cement pastes using various rheological models for different test geometry, gap and surface friction", Cement Concrete Res. 34, 11 (2004) 1993-2007.

[7] C. Hu, F. de Larrard, "The rheology of fresh highperformance concrete", Cement Concrete Res. 26, 2 (1996) 283-294.

[8] T. Yen, C.-W. Tang, C.-S. Chang, K.-H. Chen, "Flow behavior of high strength high-performance concrete", Cement Concrete Composites, 21, 5-6 (1999) 413-424.

[9] R. I. Tanner, "Engineering rheology", Ed. rev., Oxford University Press, New York, EUA (1988). 451p.

[10] F. de Larrard, T. Sedran, "Mixture-proportioning of high-performance concrete, Cement Concrete Res. 32, 11 (2002) 1699-1704.

[11] C. K. Park, M. H. Noh, T. H. Park, "Rheological properties of cementitious materials containing mineral admixtures", Cement Concrete Res. 35, 5 (2005) 842-849.

[12] J. Golaszewski, J. Szwabowski, "Influence of superplasticizers on rheological behavior of fresh cement mortars", Cement Concrete Res. 34, 2 (2004) 235-248.

[13] M. Petrou, B. Wan, F. Gardala-Maria, V. G. Kolli, $\mathrm{K}$. Harries, "Influence of mortar rheology on aggregate settlement", ACI Mater. J. 97, 4 (2000) 479-485.

[14] A. Yahia, M. Tanimura, Y. Shimoyama, "Rheological properties of highly flowable mortar containing limestone filler - effect of powder content and w/c ratio", Cement Concrete Res. 35, 3 (2005) 532-539.

[15] J.-Y. Petit, E. Wirquin, Y. Vanhove, K. Khayat, "Yield stress and viscosity equations for mortars and selfconsolidating concrete", Cement Concrete Res. 37, 5 (2007) 655-670.

[16] M. Nehdi, S. Mindess, P.-C. Aïtcin, "Rheology of highperformance concrete: effect of ultrafine particles", Cement Concrete Res. 28, 5 (1998) 687-697.

[17] E. Moulin, P. Blanc, D. Sorrentino, "Influence of key cement chemical parameters on the properties of metakaolin blended cements", Cement Concrete Composites 23, 6 (2001) 463-469.

[18] N. Roussel, "A thixotropy model for fresh fluid concretes: theory, validation and applications", Cement Concrete Res. 36, 10 (2006) 1797-1806.

[19] I. R. de Oliveira, A. R. Studart, R. G. Pileggi, V. C. Pandolfelli, "Dispersão e empacotamento de partículas - princípios e aplicações em processamento cerâmico", Fazendo Arte Editorial, S. Paulo, SP (2000) 195p.

[20] J.-Y. Petit, K. H. Khayat, E. Wirquin, "Coupled effect of time and temperature on variations of yield value of highly flowable mortar", Cement Concrete Res. 36, 5 (2006) 832-841.

[21] S. Jiang, B.-G. Kim, P.-C. Aïtcin, "Importance of adequate soluble alkali content to ensure cement/ superplasticizer compatibility", Cement Concrete Research, 29, 1 (1999) 71-78.

[22] W. Prince, M. Espagne, P.-C. Aïtcin, "Ettringite formation: a crucial step in cement superplasticizer compatibility", Cement Concrete Res. 33, 5 (2003) 635-641. [23] V.Fernàndez-Altable, I. Casanova, "Influence of mixing sequence and superplasticizer dosage on the rheological response of cement pastes at different temperatures", Cement Concrete Res. 36, 7 (2006) 1222-1230.

[24] T. Sedran, F. de Larrard, F. Hourst, C. Contamines, "Mix design of self-compacting concrete (SCC)", Proc. Int. Rilem Conf. - Production Methods and Workability of Concrete, Paisley, Escócia (1996) 439-450.

[25] L. Struble, R. Szecsy, W.-G. Lei, G.-K. Sun, "Rheology of cement paste and concrete", Cement Concrete Aggregates 20, 2 (1998) 269-277.

[26] L. Kucharska, "Effect of products of the hydration of $\mathrm{C}_{3} \mathrm{~A}$ on rheology of clinker and cement pastes", Proc. Rheology of Fresh Cement and Concrete, London, Inglaterra (1990) 47-54.

[27] G. H. Tattersall, "Workability and quality control of concrete", E \& FN Spon, London, Inglaterra (1991) 262p.

[28] Y. Nakajima, K. Yamada, "The effect of the kind of calcium sulfate in cements on the dispersing ability of poly $\beta$-naphthalene sulfonate condensate superplasticizer", Cement Concrete Res. 34, 5 (2004) 839-844.

[29] J. Punkki, J. Golaszewski, O. E. Gjørv, "Workability loss of high-strength concrete", ACI Mater. J. 93, 5 (1996) 427-431.

[30] X. Zhang, J. Han, "The effect of ultra-fine admixture on the rheological property of cement paste", Cement Concrete Res. 30, 5 (2000) 827-830.

[31] D. A. Willians, A. W. Saak, H. M. Jennings, "The influence of mixing on the rheology of fresh cement paste", Cement, Concrete Res. 29, 9 (1999) 1491-1496.

[32] C. F. Ferraris, K. H. Obla, R. Hill, "The influence of mineral admixtures on the rheology of cement paste and concrete", Cement Concrete Res. 31, 2 (2001) 245-255.

[33] P. A. Claisse, O. Lorimer, M. Al Omari, "Workability of cement pastes", ACI Mater. J. 98, 6 (2001) 476-482.

[34] V. A. Ghio, "The rheology of fresh concrete and its effect on the shotcrete process", Tese (Dr. Eng. Civil), Universidade da Califórnia, Berkeley, EUA (1993).

[35] L. Agulló, B. Toralles-Carbonari, R. Gettu, A. Aguado, "Fluidity of cement pastes with mineral admixtures and 
superplasticizer - a study based on the Marsh cone test", Mater. Structures 32, 7 (1999) 479-485.

[36] S. Mansoutre, P. Colombet, H. Van Damme, "Water retention and granular rheological behavior of fresh $\mathrm{C}_{3} \mathrm{~S}$ paste as a function of concentration", Cement Concrete Res. 29, 9 (1999) 1441-1453.

[37] H. Uchikawa, S. Hanehara, D. Sawaki, "The role of steric repulsive force in the dispersion of cement articles in fresh paste prepared with organic admixture", Cement Concrete Res. 27, 1 (1997) 37-50.

[38] P.-C. Aïtcin, C. Jolicoeur, J. G. MacGregor, "Superplasticizers: how they work and why they occasionally don't", Concrete Int. 16, 5 (1994) 45-52.

[39] D. Bonen, S. L. Sarkar, "The superplasticizer adsorption capacity of cement pastes, pore solution composition, and parameters affecting flow loss", Cement Concrete Res. 25, 7 (1995) 1423-1434.

[40] G. H. Tattersall, "Progress in measurement of workability by two-point test", Proc. Properties of Fresh Concrete, Hanover, Alemanha (1990) 203-212.

[41] G. H. Tattersall, P. H. Baker, "The effect of vibration on the rheological properties of fresh concrete", Magazine Concrete Res. 40, 143 (1988) 79-89.

[42] G. H. Tattersall, "Effect of vibration on the rheological properties of fresh cement pastes and concretes", Proc. Rheology of Fresh Cement and Concrete, London, Inglaterra (1991) 323-338.

[43] S. Kakuta, T. Kojima, "Rheology of fresh concrete under vibration", Proc. Rheology of Fresh Cement and Concrete, London, Inglaterra (1991) 339-342.

[44] T. Roshavelov, "Prediction of fresh concrete flow behavior based on analytical model for mixture proportioning", Cement Concrete Res. 35, 5 (2005) 831835.

[45] M. R. Geiker, M. Brandl, L. N. Thrane, D. H. Bager, O. Wallevik, The effect of measuring procedure on the apparent rheological properties of self-compacting concrete, Cement Concrete Research, 32, 11 (2002) 1791-1795.

[46] P. J. L. Domone, X. Yongmo, P. F. G. Banfill,
"Developments of the two-point workability test for highperformance concrete", Magazine Concrete Res. 51, 3 (1999) 171-179.

[47] F. de Larrard et al. "Evolution of the workability of superplasticized concretes: assessment with the BTRHEOM Rheometer", Proc. Int. Rilem Conf. - Production Methods and Workability of Concrete, Paisley, Escócia (1996) 377388.

[48] F. de Larrard, C. Hu, T. Sedran, J. C. Szitkar, M. Joly, F. Claux, F. Derkx, "A new rheometer for soft-to-fluid concrete", ACI Mater. J. 94, 3 (1997) 234-243.

[49] R. G. Pileggi, "Ferramentas para o estudo e desenvolvimento de concretos refratários", Tese Dr., UFSCar, S. Carlos, SP (2001).

[50] P. Banfill, D. Beaupré, F. Chapdelaine, F. de Larrard, P. Domone, L. Nachbaur, T. Sedran, O. Wallevik, J. E. Wallevik, "Comparison of concrete rheometers: international tests at LCPC (Nantes, France) in October, 2000”, NISTIR 6819 (2001).

[51] R. G. Pileggi, V. C. Pandolfelli, A. E. Paiva, "Novel rheometer for refractory castables", Am. Ceram. Soc. Bull. 79, 1 (2000) 54-58.

[52] M. F. M. Zain, M. Safiuddin, K. M. Yusof, “A study on the properties of freshly mixed high performance concrete", Cement Concrete Res. 29, 9 (1999) 1427-1432.

[53] S. Suhr, "Interactions between sulphates minerals and $\mathrm{C}_{3} \mathrm{~A}$ in cement paste rheology", Proc. Rheology of Fresh Cement and Concrete, London, Inglaterra (1991) 37-46.

[54] O. H. Wallevik, O. E. Gjørv, "Development of a coaxial cylinders viscometer for fresh concrete", Proc. Properties of Fresh Concrete, Hanover, Alemanha (1990) 213-224.

[55] A. L. Castro, "Aplicação de conceitos reológicos na tecnologia dos concretos de alto desempenho", Tese Dr., Universidade de S. Paulo, S. Carlos, SP (2007).

[56] K. Hattori, K. Izumi, "A new viscosity equation for nonNewtonian suspensions and its application", Proc. Rheology of Fresh Cement and Concrete, London, Inglaterra (1991) 83-92.

(Rec. 13/12/2008, Ac. 13/02/2009) 